\title{
EFFICIENT AND PRACTICAL SYNTHESIS OF 4(5)-
}

\section{ARYL-1 $H$-IMIDAZOLES AND 2,4(5)-DIARYL-1H-}

\author{
IMIDAZOLES VIA HIGHLY SELECTIVE \\ PALLADIUM-CATALYZED ARYLATION
}

REACTIONS

Fabio Bellina,* Silvia Cauteruccio, and Renzo Rossi*

SUPPORTING INFORMATION

General methods S2

Experimental details S2-S6

References S7

${ }^{1}$ H-NMR Spectra S8-S14 


\section{Experimental Section}

General Methods. Melting points are uncorrected. Precoated Merck $60 \mathrm{~F}_{254}$ aluminum silica gel sheets were used for TLC analyses. GLC analyses were performed using two types of capillary columns: an Alltech AT-35 bonded FSOT column $(30 \mathrm{~m} \times 0.25 \mathrm{~mm}$ i.d. $)$ and an Alltech AT-5 bonded FSOT column $(30 \mathrm{~m} \times 0.25 \mathrm{~mm}$ i.d.). Purifications by MPLC were performed using silica gel Merck 60 grade $9385,60 \AA$. NMR spectra were recorded at room temperature at 200 or $300 \mathrm{MHz}\left({ }^{1} \mathrm{H}\right)$ and 50.3 or $75.4 \mathrm{MHz}\left({ }^{13} \mathrm{C}\right)$ and were referred to TMS. All reactions were performed in flame-dried glassware under argon, by standard syringe, cannula and septa techniques. 4(5)-Phenyl- $1 H$-imidazole (1a), 4(5)bromo-1H-imidazole (7), phenylboronic acid (8a), 4-chlorophenylboronic acid (8b), 4methoxyphenylboronic acid (8c), 4-acetylboronic acid (8d), 2-naphthylboronic acid (8e), 2,5dimethoxyphenylboronic acid (8f), benzo[d][1,3]dioxol-5-ylboronic acid (8g), 2-formylboronic acid (8h), bis(triphenylphosphine)palladium dichloride, [1,1'-bis(diphenylphosphino)ferrocene]dichloro palladium, palladium acetate, copper(I) iodide, 4-iodoanisole (9a), 4-bromoanisole (9b), 4iodobenzotrifluoride $\quad(\mathbf{9 c}), \quad$ 4-bromonitrobenzene $\quad(\mathbf{9 d}), \quad 3,4,5$-(trimethoxy)iodobenzene $\quad(\mathbf{9 e}), \quad 2$ bromonaphthalene (9f) and ethyl 4-bromobenzoate (9g) were commercially available. 2-(4Methoxyphenyl)-4(5)-phenyl-1H-imidazole (2a) was prepared according to the protocol reported in the literature. $^{1}$

\section{Experimental details}

4(5)-Phenyl-1H-imidazole (1a). The crude reaction product obtained on entry 3 of Table 1 by Pdcatalyzed Suzuki-Miyaura reaction of $\mathbf{7}$ with 8a was purified by MPLC on silica gel with a mixture of $\mathrm{CH}_{2} \mathrm{Cl}_{2}$ and methanol (95:5) as eluent to give $\mathbf{1 a}\left(95 \mathrm{mg}\right.$, 66\%) as a colorless solid: $\mathrm{mp} 130-132{ }^{\circ} \mathrm{C}$ (lit. ${ }^{2}$ mp 131-132 $\left.{ }^{\circ} \mathrm{C}\right) .{ }^{1} \mathrm{H}$ NMR (300 MHz, $\left.\mathrm{CDCl}_{3}\right) \delta 11.70(\mathrm{~s}, 1 \mathrm{H}), 7.69(\mathrm{~m}, 3 \mathrm{H}), 7.31(\mathrm{~m}, 4 \mathrm{H}) ;{ }^{13} \mathrm{C} \mathrm{NMR}$ (75.4 MHz, $\mathrm{CDCl}_{3}$ ) $\delta$ 138.6, 135.7, 132.9, 128.8 (2C), 127.0, 125.0 (2C), 115.6; EI-MS m/z 145 (11), 
144 (100), 143 (10), 117 (17), 90 (20). Anal. Calcd. for $\mathrm{C}_{9} \mathrm{H}_{8} \mathrm{~N}_{2}: \mathrm{C}, 74.98 ; \mathrm{H}, 5.59 ; \mathrm{N}, 19.43$. Found: C, 74.77; H, 5.55; N, 19.24. 4(5)-Phenyl-1H-imidazole (1a) so obtained had spectral data in good agreement with those of commercially available 1a. GLC analysis showed that 1a was chemically pure.

4(5)-(4-Chlorophenyl)-1H-imidazole (1b). The crude reaction product obtained in entry 4 of Table 1 by Pd-catalyzed Suzuki-Miyaura reaction of $\mathbf{7}$ with $\mathbf{8 b}$ was purified by MPLC on silica gel with a mixture of $\mathrm{CH}_{2} \mathrm{Cl}_{2}$ and methanol $(95: 5)$ as eluent to give $\mathbf{1 b}(0.144 \mathrm{~g}, 82 \%)$ as a pale pink solid: $\mathrm{mp}$ 120-123ㅇ ${ }^{\circ}$ (lit. $\left.{ }^{2} \mathrm{mp} 140-143^{\circ} \mathrm{C}\right) ;{ }^{1} \mathrm{H}$ NMR $\left(200 \mathrm{MHz}, \mathrm{CDCl}_{3}\right) \delta 11.5$ (s, 1H), 7.73 (br s, 1H), 7.63 (d, J $=8.4 \mathrm{~Hz}, 2 \mathrm{H}), 7.31(\mathrm{~m}, 3 \mathrm{H}) ;{ }^{13} \mathrm{C} \mathrm{NMR}\left(50.3 \mathrm{MHz}, \mathrm{CDCl}_{3}\right) \delta 138.1,135.7,132.8,131.4,128.9(2 \mathrm{C})$, 126.3 (2C), 115.1; EI-MS m/z 180 (32), 179 (12), 178 (100), 151 (12), 116 (14). Anal. Calcd. for $\mathrm{C}_{9} \mathrm{H}_{7} \mathrm{ClN}_{2}: \mathrm{C}, 60.52 ; \mathrm{H}, 3.95 ; \mathrm{N}, 15.68$. Found: $\mathrm{C}, 60.49 ; \mathrm{H}, 3.87 ; \mathrm{N}, 15.33$. GLC analysis showed that 1b was chemically pure.

4(5)-(4-Methoxyphenyl)-1H-imidazole (1c). The crude reaction product obtained in entry 5 of Table 1 by Pd-catalyzed Suzuki-Miyaura reaction of 7 with $8 \mathbf{c}$ was purified by MPLC on silica gel with a mixture of $\mathrm{CH}_{2} \mathrm{Cl}_{2}$ and methanol $(95: 5)$ as eluent to give 1c $(0.158 \mathrm{~g}, 91 \%)$ as a yellow solid: mp $113-$ $115^{\circ} \mathrm{C}$ (lit. $\left.{ }^{3} \mathrm{mp} 136-138^{\circ} \mathrm{C}\right) ;{ }^{1} \mathrm{H}$ NMR $\left(200 \mathrm{MHz}, \mathrm{CDCl}_{3}\right) \delta 11.28$ (br s, 1H), 7.63 (m, 3H), $7.25(\mathrm{~d}, \mathrm{~J}=$ $2.2 \mathrm{~Hz}, 1 \mathrm{H}), 6.90(\mathrm{~m}, 2 \mathrm{H}), 3.80(\mathrm{~s}, 3 \mathrm{H}) ;{ }^{13} \mathrm{C} \mathrm{NMR}\left(50.3 \mathrm{MHz}, \mathrm{CDCl}_{3}\right) \delta 158.8,138.2,135.3,126.3$ (2C), 125.7, 114.7, 114.2 (2C), 55.3; EI-MS m/z 175 (11), 174 (100), 159 (61), 131 (27), 77 (11). Anal. Calcd. for $\mathrm{C}_{10} \mathrm{H}_{10} \mathrm{~N}_{2} \mathrm{O}: \mathrm{C}, 68.95 ; \mathrm{H}, 5.79 ; \mathrm{N}, 16.08$. Found: C, 68.85; H, 5.71; N, 15.92. GLC analysis showed that 1c, which had ${ }^{1} \mathrm{H}$ NMR data in agreement with those previously reported, ${ }^{3}$ was chemically pure.

4(5)-(4-Acetylphenyl)-1H-imidazole (1d). The crude reaction product obtained in entry 6 of Table 1 by Pd-catalyzed Suzuki-Miyaura reaction of $\mathbf{7}$ with 8d was purified by MPLC on silica gel with a mixture of $\mathrm{CH}_{2} \mathrm{Cl}_{2}$ and methanol $(95: 5)$ as eluent to give $\mathbf{1 d}(0.154 \mathrm{~g}, 83 \%)$ as a pale pink solid: $\mathrm{mp} 171-174^{\circ} \mathrm{C}$; ${ }^{1} \mathrm{H}$ NMR $\left(200 \mathrm{MHz}, \mathrm{CD}_{3} \mathrm{OD}\right) \delta 8.00(\mathrm{~m}, 2 \mathrm{H}), 7.85(\mathrm{~m}, 3 \mathrm{H}), 7.62(\mathrm{~s}, 1 \mathrm{H}), 2.59(\mathrm{~s}, 3 \mathrm{H}) ;{ }^{13} \mathrm{C}$ NMR $(50.3$ 
$\left.\mathrm{MHz}, \mathrm{CD}_{3} \mathrm{OD}\right) \delta$ 199.0, 138.7, 136.6, 134.4, 130.2 (2C), 128.3, 125.7 (2C), 118.5, 26.6; EI-MS m/z 175

(11), 174 (100), 159 (61), 131 (27), 77 (11). Anal. Calcd. for $\mathrm{C}_{11} \mathrm{H}_{10} \mathrm{~N}_{2} \mathrm{O}$ : C, 70.95; H, 5.41; N, 15.04.

Found: C, 70.87; H, 5.37; N, 14.91. GLC analysis showed that 1d had chemical purity higher than $99 \%$.

4(5)-(2-Naphthyl)-1H-imidazole (1e). The crude reaction product obtained in entry 7 of Table 1 by Pdcatalyzed Suzuki-Miyaura reaction of $\mathbf{7}$ with $\mathbf{8 e}$ was purified by MPLC on silica gel with a mixture of $\mathrm{CH}_{2} \mathrm{Cl}_{2}$ and methanol (95:5) as eluent to give 1e $(0.174 \mathrm{~g}, 90 \%)$ as a brown solid: $\mathrm{mp} 165-166^{\circ} \mathrm{C}\left(\mathrm{lit}^{4}\right.$ mp $\left.171{ }^{\circ} \mathrm{C}\right) ;{ }^{1} \mathrm{H}$ NMR $\left(200 \mathrm{MHz}, \mathrm{CD}_{3} \mathrm{OD}\right) \delta 8.18(\mathrm{~s}, 1 \mathrm{H}), 7.82(\mathrm{~m}, 4 \mathrm{H}), 7.54(\mathrm{~s}, 1 \mathrm{H}), 7.43(\mathrm{~m}, 3 \mathrm{H}) ;{ }^{13} \mathrm{C}$ NMR (50.3 MHz, CD 3 OD) $\delta 139.6,137.3,135.2,134.0,131.7,129.4,129.0,128.7,127.3,126.6,124.7$, 123.8, 117.2; EI-MS m/z 196 (62), 195 (100), 194 (62), 167 (23), 139 (42). Anal. Calcd. for $\mathrm{C}_{13} \mathrm{H}_{10} \mathrm{~N}_{2}$ : C, 80.39; H, 5.19; N, 14.42. Found: C, 80.31; H, 5.08; N, 14.35. GLC analysis showed that 1e had chemical purity higher than $98 \%$.

4(5)-(Benzo[d][1,3]-dioxol-5-yl)-1H-imidazole (1g). The crude reaction product obtained in entry 9 of Table 1 by Pd-catalyzed Suzuki-Miyaura reaction of $\mathbf{7}$ with $\mathbf{8 g}$ was purified by MPLC on silica gel with a mixture of $\mathrm{CH}_{2} \mathrm{Cl}_{2}$ and methanol (93:7) as eluent to give $1 \mathrm{~g}(0.162 \mathrm{~g}, 86 \%)$ as a red solid: $\mathrm{mp} 98-99^{\circ} \mathrm{C}$ $\left(\mathrm{lit}^{5} \mathrm{mp} 100-101^{\circ} \mathrm{C}\right) ;{ }^{1} \mathrm{H} \mathrm{NMR}\left(200 \mathrm{MHz}, \mathrm{CD}_{3} \mathrm{OD}\right) \delta 7.68(\mathrm{~d}, \mathrm{~J}=0.8 \mathrm{~Hz}, 1 \mathrm{H}), 7.28(\mathrm{~d}, \mathrm{~J}=0.8 \mathrm{~Hz}, 1 \mathrm{H})$, $7.18(\mathrm{~m}, 2 \mathrm{H}), 6.81(\mathrm{~m}, 1 \mathrm{H}), 5.93(\mathrm{~s}, 2 \mathrm{H}) ;{ }^{13} \mathrm{C}$ NMR $\left(50.3 \mathrm{MHz}, \mathrm{CD}_{3} \mathrm{OD}\right) \delta 149.5,148.1,139.5,136.7$, 128.6, 119.4, 115.9, 109.4, 106.6, 102.3; EI-MS m/z 189 (12), 188 (100), 187 (31), 130 (8), 103 (14). Anal. Calcd. for $\mathrm{C}_{10} \mathrm{H}_{8} \mathrm{~N}_{2} \mathrm{O}_{2}$ : C, 63.82; H, 4.28; N, 14.89. Found: C, 63.77; H, 4.19; N, 14.74. GLC analysis showed that $\mathbf{1 g}$ had chemical purity higher than $98 \%$.

4(5)-Phenyl-2-(4-nitrophenyl)-1H-imidazole (2c). The crude reaction product obtained in entry 4 of Table 2 by Pd-catalyzed and Cu-mediated arylation of 1a with 9d was purified by MPLC on silica gel with a mixture of toluene and $\operatorname{EtOAc}\left(70: 30+0.1 \% \mathrm{Et}_{3} \mathrm{~N}\right)$ as eluent to give $2 \mathrm{c}(0.207 \mathrm{~g}, 78 \%)$ as an orange solid: $\mathrm{mp} 172-173^{\circ} \mathrm{C}\left(\mathrm{lit}^{6} \mathrm{mp} 183^{\circ} \mathrm{C}\right) ;{ }^{1} \mathrm{H}$ NMR $\left(200 \mathrm{MHz}, \mathrm{DMSO}-\mathrm{d}_{6}\right) \delta 8.31(\mathrm{~m}, 4 \mathrm{H}), 7.91(\mathrm{~m}$, 3H), $7.36(\mathrm{~m}, 3 \mathrm{H}) ;{ }^{13} \mathrm{C}$ NMR (50.3 MHz, DMSO-d $\left.{ }_{6}\right) \delta 146.4,143.9,142.3,136.3,134.1,128.5$ (2C), 
126.6, 125.5 (2C), 124.5 (2C), 124.2 (2C), 116.2; EI-MS m/z 266 (18), 265 (100), 235 (7), 219 (31), 218

(8). Anal. Calcd. for $\mathrm{C}_{15} \mathrm{H}_{11} \mathrm{~N}_{3} \mathrm{O}_{2}$ : C, 67.92; H, 4.18; N, 15.84. Found: C, 67.81; H, 4.03; N, 15.71. GLC analysis showed that $\mathbf{2 c}$ had chemical purity higher than $98 \%$.

4(5)-Phenyl-2-[(3,4,5-trimethoxy)phenyl]-1H-imidazole (2d). The crude reaction product obtained in entry 5 of Table 2 by Pd-catalyzed and Cu-mediated arylation of 1a with 9 e was purified by MPLC on silica gel with a mixture of $\mathrm{CH}_{2} \mathrm{Cl}_{2}$ and methanol (98:2) as eluent to give 2d $(0.177 \mathrm{~g}, 57 \%)$ as a colorless solid: mp 127-128 ${ }^{\circ}$; ${ }^{1} \mathrm{H}$ NMR $\left(200 \mathrm{MHz}, \mathrm{CDCl}_{3}\right) \delta 7.72(\mathrm{~m}, 2 \mathrm{H}), 7.41(\mathrm{~s}, 1 \mathrm{H}), 7.33(\mathrm{~m}, 3 \mathrm{H})$, $7.14(\mathrm{~s}, 2 \mathrm{H}), 3.79$ (s, 3H), $3.58(\mathrm{~s}, 6 \mathrm{H}) ;{ }^{13} \mathrm{C} \mathrm{NMR}\left(50.3 \mathrm{MHz}, \mathrm{CDCl}_{3}\right) \delta 153.5(2 \mathrm{C}), 147.6,144.2,138.6$, 131.8, 128.9 (2C), 127.4, 125.2, 125.1 (2C), 118.2, 103.0 (2C), 60.9, 55.9 (2C); EI-MS m/z 311 (20), 310 (100), 296 (10), 295 (52), 237 (10). Anal. Calcd. for $\mathrm{C}_{18} \mathrm{H}_{18} \mathrm{~N}_{2} \mathrm{O}_{3}$ : C, 69.66; H, 5.85; N, 9.03. Found: C, 69.57; H, 5.79; N, 8.94. GLC analysis showed that 2d had chemical purity higher than 99\%.

2-(2-Naphthyl)-4(5)-phenyl-1H-imidazole (2e). The crude reaction product obtained in entry 6 of Table 2 by Pd-catalyzed and Cu-mediated arylation of 19 with $9 \mathbf{f}$ was purified by MPLC on silica gel with a mixture of toluene and EtOAc $\left(80: 20+0.1 \% \mathrm{Et}_{3} \mathrm{~N}\right)$ as eluent to give $2 \mathrm{e}(0.167 \mathrm{~g}, 62 \%)$ as a light brown solid: mp $196-197^{\circ} \mathrm{C}\left(\mathrm{lit}^{7} \mathrm{mp} 194-198^{\circ} \mathrm{C}\right) ;{ }^{1} \mathrm{H}$ NMR $\left(200 \mathrm{MHz}, \mathrm{DMSO}-\mathrm{d}_{6}\right) \delta 8.57$ (s, 1H), 8.23 $(\mathrm{m}, 1 \mathrm{H}), 7.94(\mathrm{~m}, 6 \mathrm{H}), 7.50(\mathrm{~m}, 4 \mathrm{H}), 7.27(\mathrm{~m}, 1 \mathrm{H}) ;{ }^{13} \mathrm{C}$ NMR $\left(50.3 \mathrm{MHz}, \mathrm{DMSO}-\mathrm{d}_{6}\right) \delta$ 146.1, 140.5, $132.9,132.6,128.5$ (2C), 128.2, 128.0 (2C), 127.7, 126.6 (2C), 126.3, 126.2, $124.4(2 \mathrm{C}), 123.3,123.0$, 114.8; EI-MS m/z, 271 (21), 270 (100), 269 (20), 207 (10), 139 (10). Anal. Calcd. for $\mathrm{C}_{19} \mathrm{H}_{14} \mathrm{~N}_{2}: \mathrm{C}_{\text {, }}$ 84.42; H, 5.22; N, 10.36. Found: C, 84.37; H, 5.18; N, 10.27. GLC analysis showed that 2e was chemically pure.

2-[(4-Ethoxycarbonyl)phenyl]-4(5)-(2-naphthyl)-1H-imidazole (2f). The crude reaction product obtained in entry 7 of Table 2 by Pd-catalyzed and $\mathrm{Cu}$-mediated arylation of $\mathbf{1 e}$ with $\mathbf{9 g}$ was purified by MPLC on silica gel with a mixture of toluene and EtOAc $\left(70: 30+0.1 \% \mathrm{Et}_{3} \mathrm{~N}\right)$ as eluent to give $2 \mathbf{f}$ $(0.137 \mathrm{~g}, 40 \%)$ as a highly fluorescent, light brown solid: $\mathrm{mp} 183-184{ }^{\circ} \mathrm{C} ;{ }^{1} \mathrm{H}$ NMR $(200 \mathrm{MHz}$, DMSO- 
$\left.\mathrm{d}_{6}\right) \delta 8.43(\mathrm{~s}, 1 \mathrm{H}), 8.22(\mathrm{~m}, 2 \mathrm{H}), 8.00(\mathrm{~m}, 7 \mathrm{H}), 7.51(\mathrm{~m}, 2 \mathrm{H}), 4.37(\mathrm{q}, \mathrm{J}=7.0 \mathrm{~Hz}, 2 \mathrm{H}), 1.35(\mathrm{t}, \mathrm{J}=7.0$

$\mathrm{Hz}, 3 \mathrm{H}) ;{ }^{13} \mathrm{C}$ NMR (50.3 MHz, DMSO-d 6 ) $\delta$ 165.4, 147.6, 140.5, 134.5, 133.4, 132.0, 129.7 (2C), 128.9

(2C), 127.9, 127.7, 127.5 (2C), 126.3, 125.3, 124.9 (2C), 123.6, 122.0, 60.7, 14.1; EI-MS m/z 343 (25),

342 (100), 315 (7), 314 (31), 269 (8), 139 (17). Anal. Calcd. for $\mathrm{C}_{22} \mathrm{H}_{18} \mathrm{~N}_{2} \mathrm{O}_{2}$ : C, 77.17; H, 5.30; N, 8.18.

Found: C, 77.09; H, 5.25; N, 8.10. GLC analysis showed that $\mathbf{2 f}$ had chemical purity higher than $98 \%$.

5-(4-Methoxyphenyl)-2-(4-(trifluoromethyl)phenyl)-1H-imidazole (2g). The crude reaction product

obtained in entry 8 of Table 2 by Pd-catalyzed and $\mathrm{Cu}$-mediated arylation of $\mathbf{1 c}$ with $\mathbf{9 c}$ was purified by MPLC on silica gel with a mixture of toluene and EtOAc $\left(80: 20+0.1 \% \mathrm{Et}_{3} \mathrm{~N}\right)$ as eluent to give $\mathbf{2 g}$ $(0.119 \mathrm{~g}, 42 \%)$ as a highly fluorescent, yellow solid: $\mathrm{mp} 187-189^{\circ} \mathrm{C} ;{ }^{1} \mathrm{H}$ NMR $\left(200 \mathrm{MHz}, \mathrm{DMSO}-\mathrm{d}_{6}\right) \delta$ $8.23(\mathrm{~m}, 2 \mathrm{H}), 7.82(\mathrm{~m}, 4 \mathrm{H}), 7.70(\mathrm{~s}, 1 \mathrm{H}), 6.99(\mathrm{~m}, 2 \mathrm{H}), 3.80 \mathrm{ppm}(\mathrm{s}, 3 \mathrm{H}) ;{ }^{13} \mathrm{C}$ NMR $(50.3 \mathrm{MHz}, \mathrm{DMSO}-$ $\left.\mathrm{d}_{6}\right) \delta 158.2,144.1,134.1,128.1,127.5,126.9,125.8(2 \mathrm{C}), 125.7(2 \mathrm{C}), 125.6,125.2(2 \mathrm{C}), 116.9,113.9$ (2C), 55.0; EI-MS m/z 319 (19), 318 (100), 304 (9), 303 (51), 275 (12). Anal. Calcd. for $\mathrm{C}_{17} \mathrm{H}_{13} \mathrm{~F}_{3} \mathrm{~N}_{2} \mathrm{O}$ : C, 64.15; H, 4.12; N, 8.80. Found: C, 64.11; H, 4.10; N, 8.73. GLC analysis showed that $2 \mathrm{~g}$ had chemical purity higher than $99 \%$.

5-(4-Chlorophenyl)-2-(4-(trifluoromethyl)phenyl)-1H-imidazole $(2 \mathrm{~h})$. The crude reaction product obtained in entry 9 of Table 2 by Pd-catalyzed and Cu-mediated arylation of $\mathbf{1 b}$ with $\mathbf{9 c}$ was purified by MPLC on silica gel with a mixture of toluene and EtOAc $\left(90: 10+0.1 \% \mathrm{Et}_{3} \mathrm{~N}\right)$ as eluent to give $2 \mathbf{h}$ $(0.155 \mathrm{~g}, 48 \%)$ as a highly fluorescent, yellow solid: $\mathrm{mp} 163-165^{\circ} \mathrm{C} ;{ }^{1} \mathrm{H}$ NMR $\left(200 \mathrm{MHz}, \mathrm{DMSO}-\mathrm{d}_{6}\right) \delta$ $8.23(\mathrm{~m}, 2 \mathrm{H}), 7.89(\mathrm{~m}, 5 \mathrm{H}), 7.47 \mathrm{ppm}(\mathrm{m}, 2 \mathrm{H}) ;{ }^{13} \mathrm{C}$ NMR $\left(50.3 \mathrm{MHz}, \mathrm{DMSO}-\mathrm{d}_{6}\right) \delta 144.7,133.9,132.5$, 130.8 (2C), 128.8, 128.5 (2C), 128.1, 127.8, 126.1 (2C), 125.8, 125.7, 125.4 (2C); EI-MS m/z 324 (33), 323 (19), 322 (100), 185 (6), 116 (6), 89 (10). Anal. Calcd. for $\mathrm{C}_{16} \mathrm{H}_{10} \mathrm{ClF}_{3} \mathrm{~N}_{2}$ : C, 59.55; H, 3.12; N, 8.68. Found: C, 59.59; H, 3.08; N, 8.56. GLC analysis showed that $\mathbf{2 h}$ had chemical purity higher than $98 \%$. 


\section{REFERENCES}

(1) Bellina, F.; Calandri, C.; Cauteruccio, S.; Rossi, R. Tetrahedron 2007, 63, 1970.

(2) Ten Have, R.; Huisman, M.; Meetsma, A.; van Leusen, A. M. Tetrahedron 1997, 53, 11355.

(3) Horne, D. A.; Yakushijin, K.; Büchi, G. Heterocycles 1994, 39, 139.

(4) Voets, M.; Antes, I.; Scherer, C.; Muller-Vieira, U.; Biemel, K.; Barassin, C.; MarchaisOberwinkler, S.; Hartmann, R. W. J. Med. Chem. 2005, 48, 6632.

(5) Wilkinson, C. F.; Hetnarski, K.; Hicks, L. Pest. Biochem. Physiol. 1973, 4, 299.

(6) Bunge, K.; Huisgen, R.; Raab, R.; Sturm, H. J. Chem. Ber. 1972, 105, 1307.

(7) Hirohiko, H.; Yoshimasa, K.; Takayuki, M. PCT Int. Appl. 2005, WO 121101. 
${ }^{1} \mathrm{H}-\mathrm{NMR}\left(200 \mathrm{MHz}, \mathrm{CD}_{3} \mathrm{OD}\right.$ )
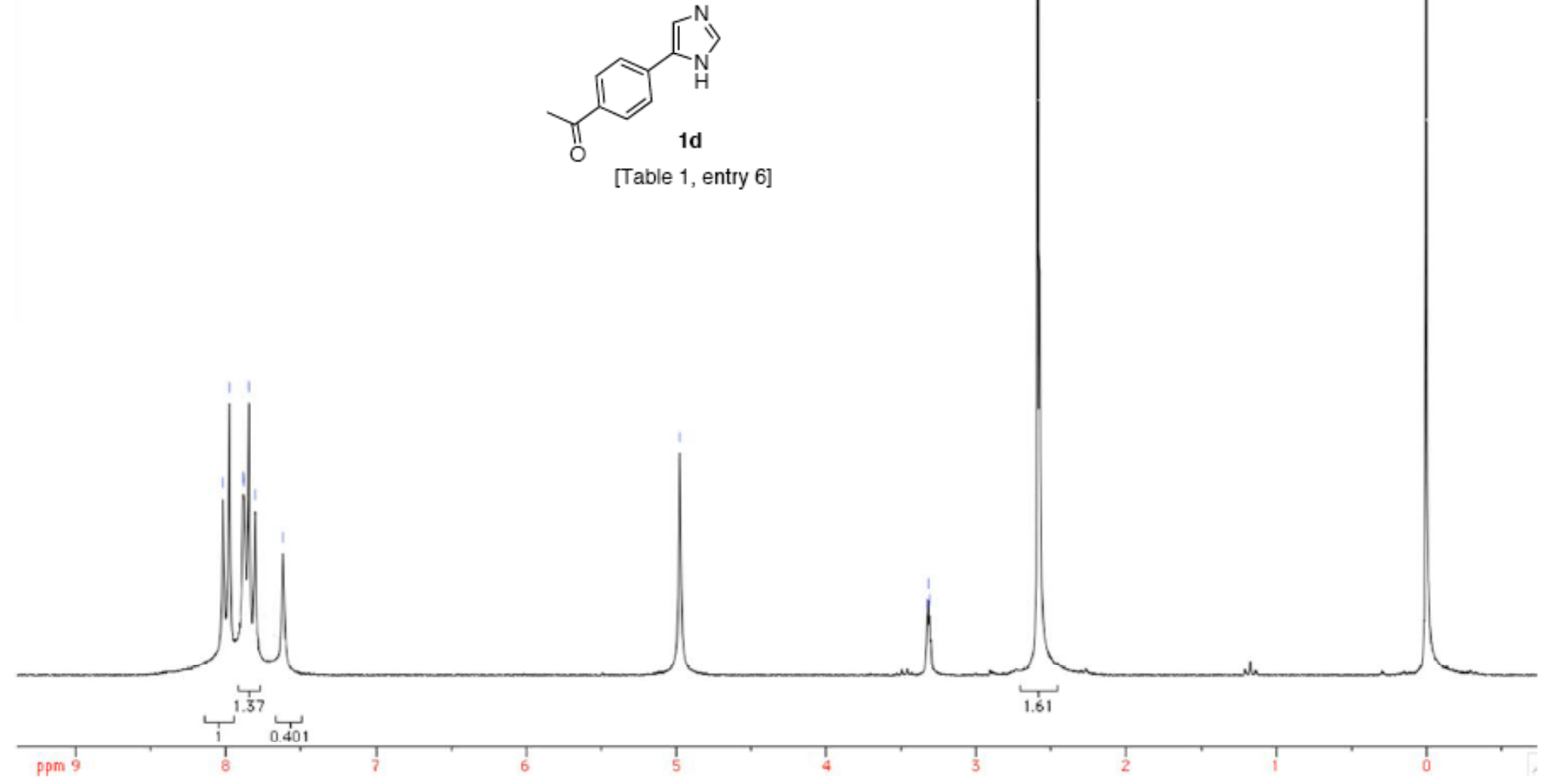


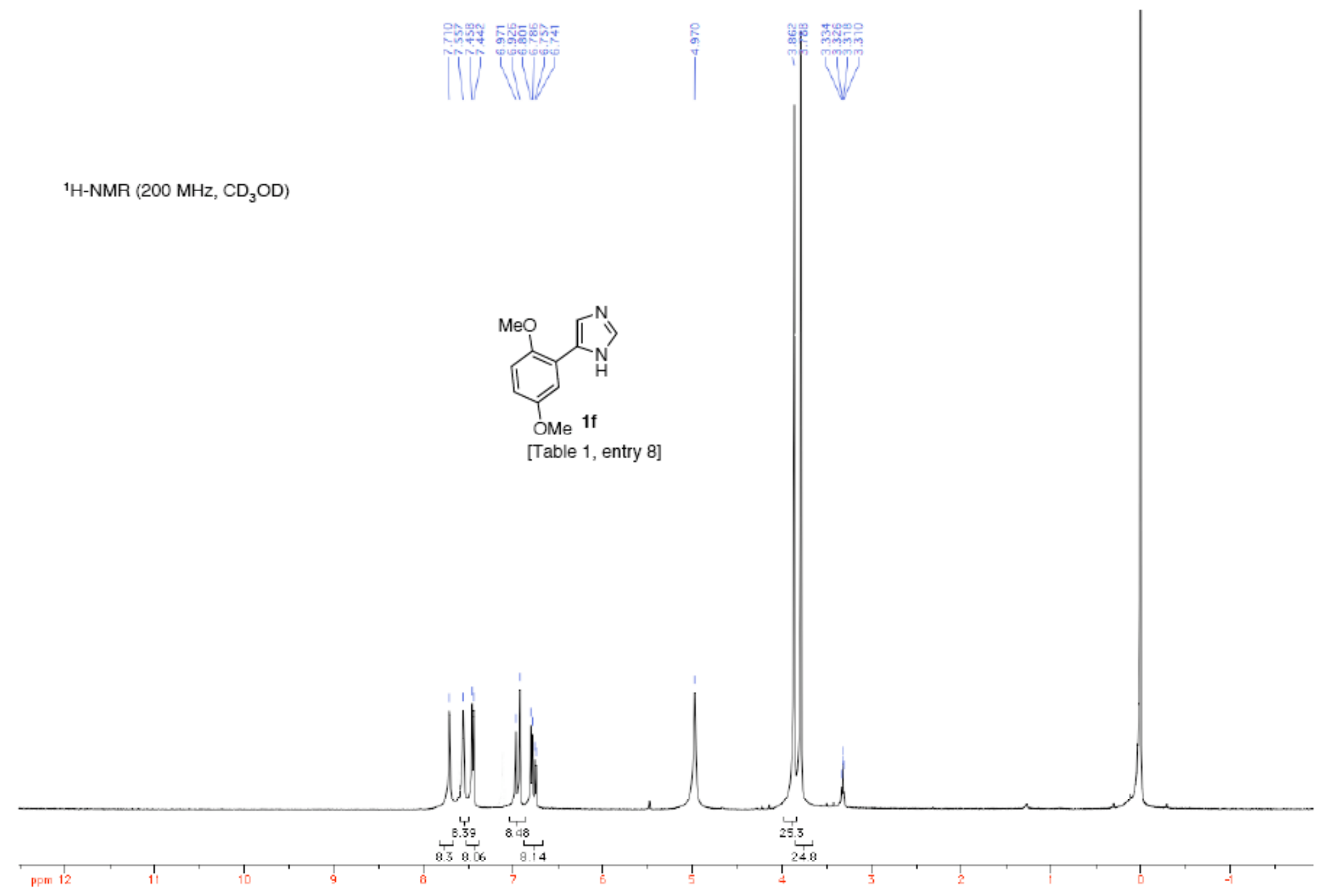


1'H-NMR (200 MHz, CDCl $)$

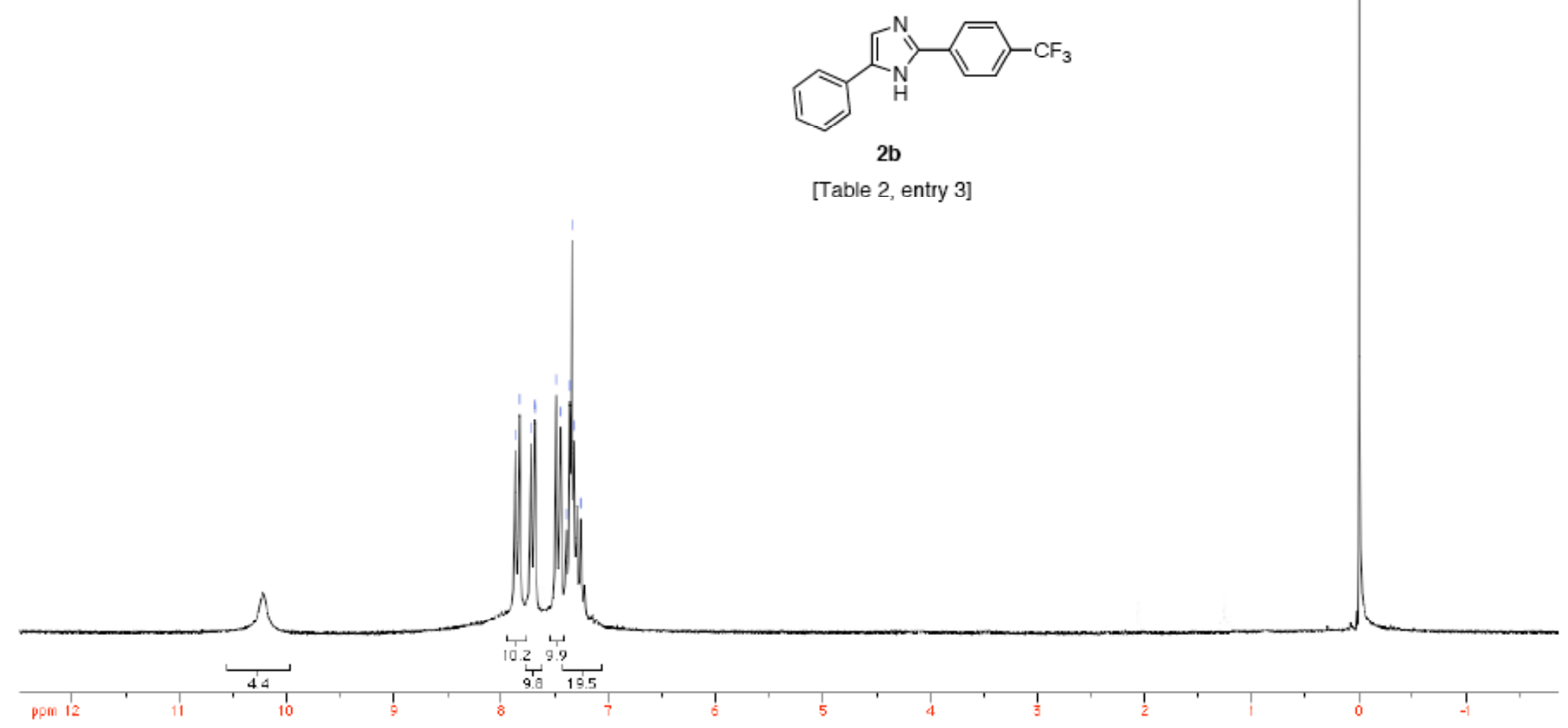


${ }^{1} \mathrm{H}-\mathrm{NMR}\left(200 \mathrm{MHz}, \mathrm{CDCl}_{3}\right)$

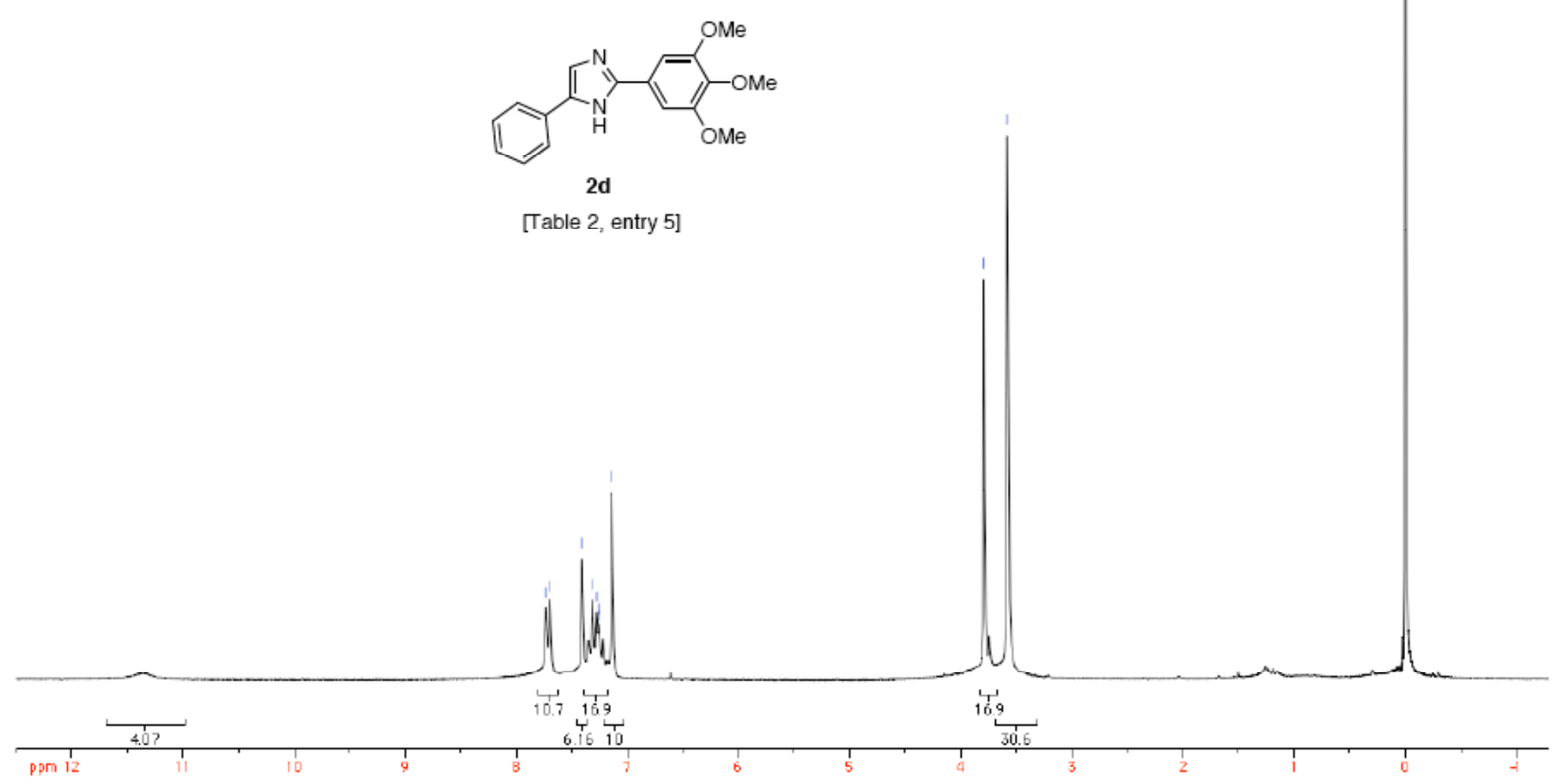


${ }^{1} \mathrm{H}-\mathrm{NMR}\left(200 \mathrm{MHz}\right.$, DMSO-d $\left.{ }_{6}\right)$

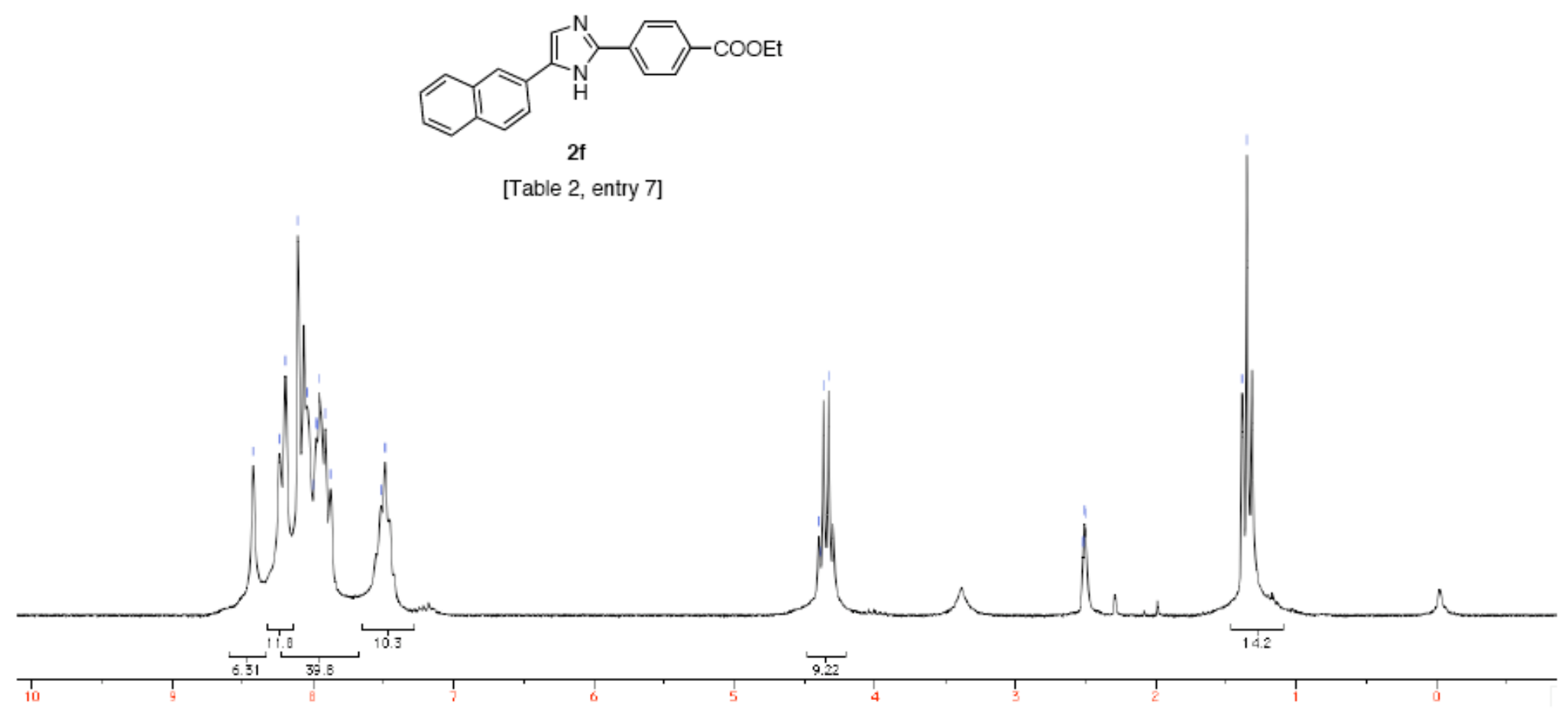




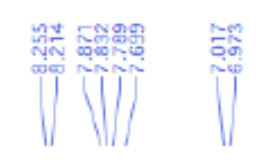

${ }^{1} \mathrm{H}-\mathrm{NMR}\left(200 \mathrm{MHz}\right.$, DMSO-d ${ }_{6}$ )

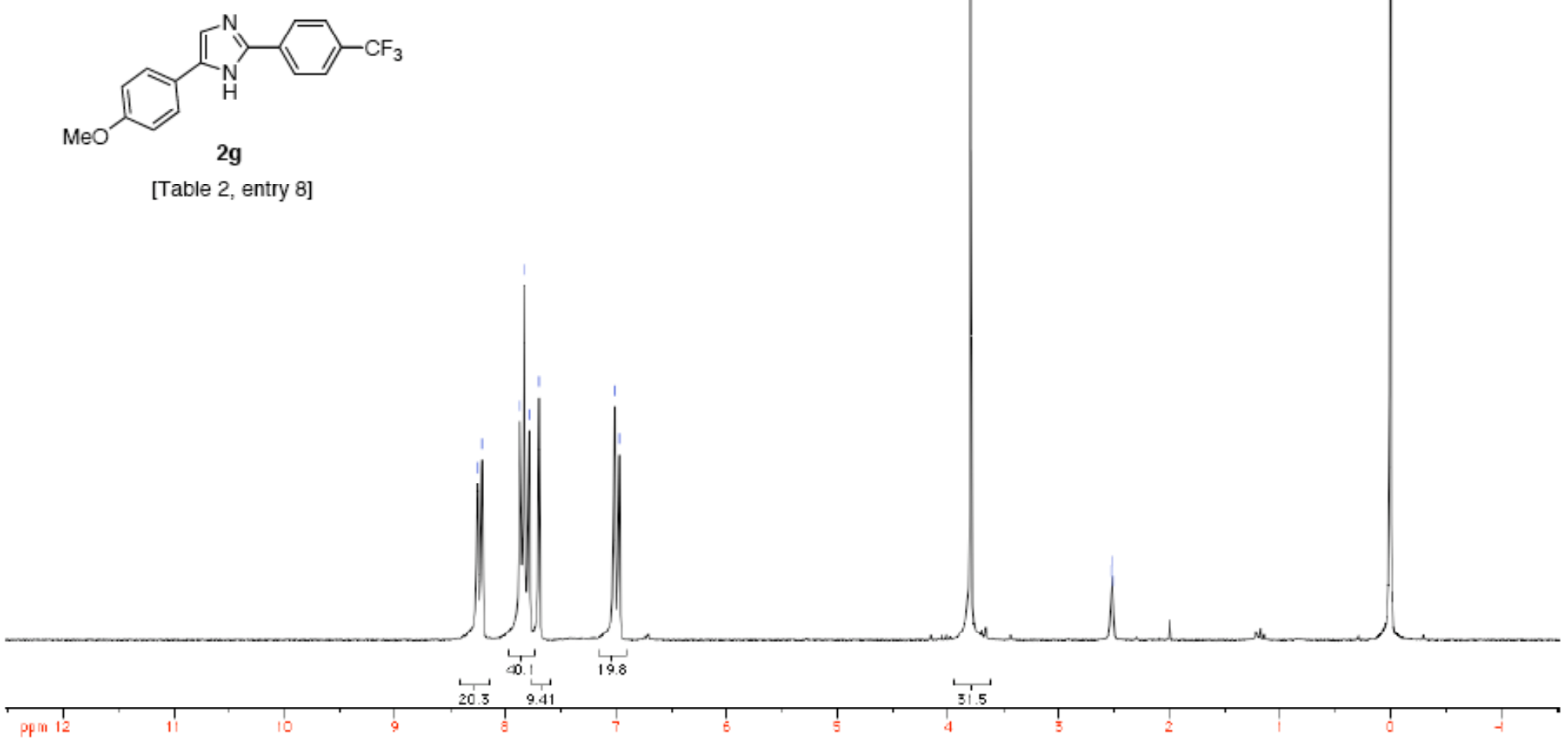


${ }^{1} \mathrm{H}-\mathrm{NMR}\left(200 \mathrm{MHz}\right.$, DMSO-d ${ }_{6}$ )

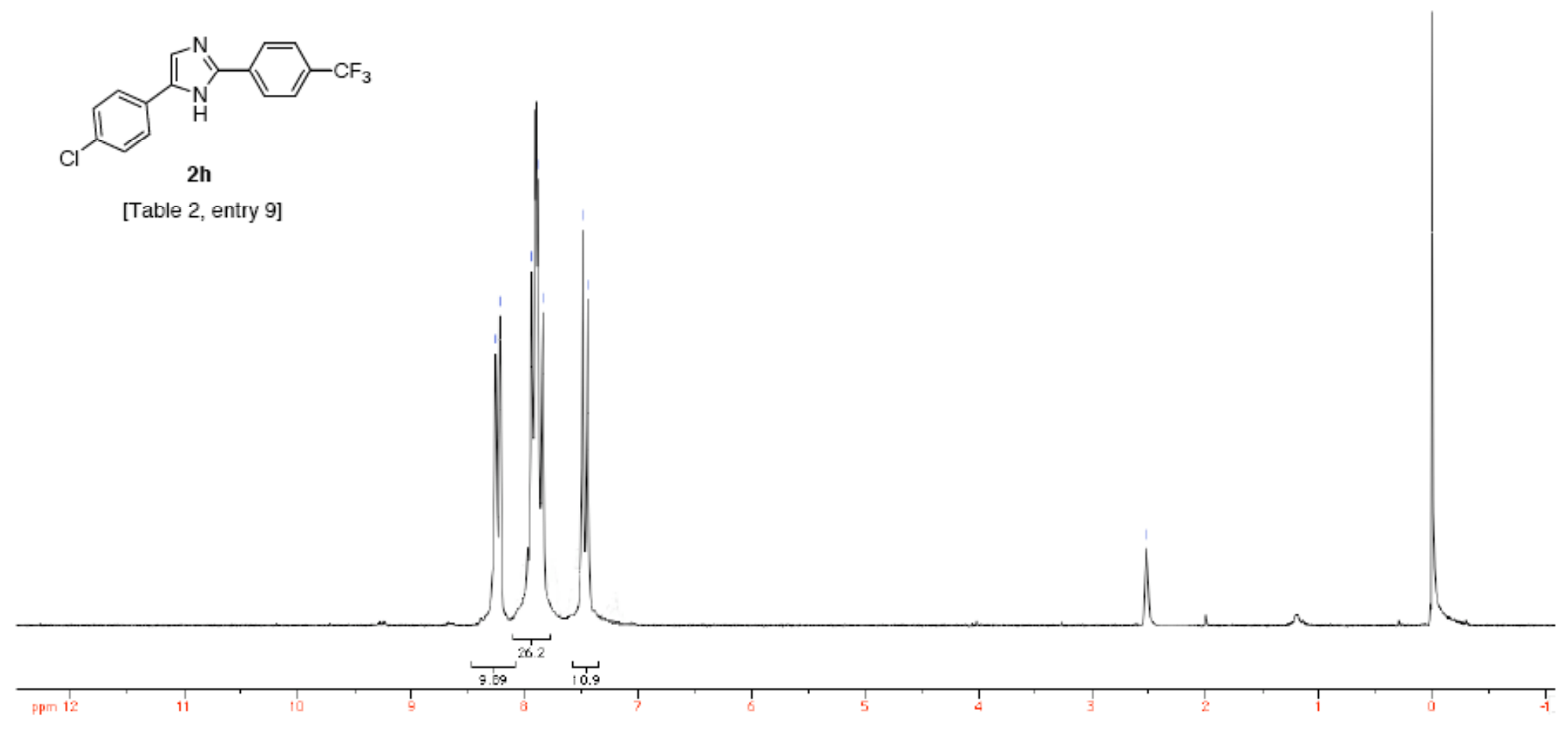

